\title{
Outbreak of severe acute respiratory syndrome in Hong Kong Special Administrative Region: case report
}

\author{
Moira Chan-Yeung, W C Yu
}

Division of

Respiratory and

Critical Care

Medicine, 4/F,

Professorial Block,

Queen Mary

Hospital, University

of Hong Kong

Hong Kong, SAR,

China

Moira Chan-Yeung

professor

Department of

Medicine, Princess

Margaret Hospital,

Hong Kong, SAR,

China

W C Yu

consultant physician

Correspondence to:

M Chan-Yeung

mmwchan@

hkucc.hku.hk

BMJ 2003;326:850-2

\begin{abstract}
Objective To describe the outbreak of severe acute respiratory syndrome in Hong Kong.

Design Descriptive case series.

Setting Hong Kong, Special Administrative Region, China

Results The outbreak started with a visitor from southern China on 21 February. At the hospitals where the first cases were treated the disease spread quickly among healthcare workers, and then out into the community as family members became infected. By 1 April, 685 cases had been reported with 16 deaths. Symptoms include high fever and one or more respiratory symptoms (including cough, shortness of breath, and difficulty breathing). Changes in lung tissue suggest that part of the lung damage is due to cytokines induced by the microbial agent, which has led to empirical treatment with corticosteroids, broad spectrum antiviral agent, and antibacterial cover. There is strong evidence that a novel coronavirus is the pathogen. Precautions for droplet infection should be instituted, including the wearing of masks and rigorous disinfection and hygiene procedures. On 27 March the Department of Health announced drastic measures, including vigorous contact tracing and examination, quarantine of contacts in their homes, and closure of all schools and universities.

Conclusion The rapidity of the spread of the disease and the morbidity indicate that the agent responsible is highly infectious and virulent. Strict infection
\end{abstract}

Cumulative number of cases of severe acute respiratory syndrome reported to the Department of Health, Hong Kong, from 14 March to 1 April 2003

\begin{tabular}{|c|c|c|c|c|c|}
\hline \multirow[b]{2}{*}{ Date } & \multicolumn{3}{|c|}{ Healthcare workers } & \multirow[b]{2}{*}{$\begin{array}{l}\text { Patients, } \\
\text { families, and } \\
\text { visitors }\end{array}$} & \multirow[b]{2}{*}{ Cumulative total } \\
\hline & $\begin{array}{l}\text { Hospital where } \\
\text { outbreak started }\end{array}$ & $\begin{array}{c}\text { Other } \\
\text { healthcare } \\
\text { workers }\end{array}$ & Total & & \\
\hline 14 March & 3 & 0 & 3 & 0 & 3 \\
\hline 15 March & 36 & 0 & 36 & 0 & 36 \\
\hline 16 March & $36+13^{*}$ & 0 & 49 & 0 & 49 \\
\hline 17 March & $44+16^{*}$ & 12 & 72 & 23 & 95 \\
\hline 18 March & $47+17^{\star}$ & 20 & 84 & 39 & 123 \\
\hline 19 March & $54+17^{\star}$ & 21 & 92 & 58 & 150 \\
\hline 20 March & $58+17^{\star}$ & 24 & 99 & 74 & 173 \\
\hline 21 March & $66+17^{*}$ & 27 & 110 & 93 & 203 \\
\hline 22 March & $68+17^{*}$ & 29 & 114 & 108 & 222 \\
\hline 23 March & $73+17^{*}$ & 32 & 122 & 125 & 247 \\
\hline 24 March & $78+17^{*}$ & 34 & 129 & 136 & 265 \\
\hline 25 March & $82+17^{\star}$ & 35 & 134 & 156 & 290 \\
\hline 26 March & $88+18^{*}$ & 37 & 143 & 176 & 319 \\
\hline 27 March & NA & NA & 149 & 221 & 370 \\
\hline 28 March & NA & NA & 153 & 272 & 425 \\
\hline 29 March & NA & NA & 156 & 314 & 470 \\
\hline 30 March & NA & NA & 162 & 368 & 530 \\
\hline 31 March & NA & NA & 164 & 446 & 610 \\
\hline 1 April & NA & NA & 168 & 517 & 685 \\
\hline
\end{tabular}

$\mathrm{NA}=$ Not assessed. * Medical students control measures for droplet and contact transmission by healthcare workers, a vigilant healthcare profession, and public education are essential for disease prevention.

\section{Introduction}

On 12 March 2003, the World Health Organization issued a global alert on atypical pneumonia, called severe acute respiratory syndrome, after reports from the Department of Health of Hong Kong of an outbreak of pneumonia in one of its public hospitals. At about the same time, the WHO received reports of the syndrome from China, Singapore, Vietnam, Thailand, Indonesia, Taiwan, and Philippines, as well as from countries in other continents including Canada, the United States, and Germany. The disease originated in Guangdong at the end of last year and has affected over 300 people and killed five. ${ }^{1}$

We here describe the outbreak that occurred in Hong Kong Special Administrative Region.

\section{Case reports}

History of the outbreak

The outbreak in Hong Kong started when a doctor from southern China arrived on 21 February 2003 and stayed in a local hotel. He had been unwell for a few days before the trip but now became seriously ill and died in a local hospital. However, he had infected his brother-in-law, two nurses in the hospital, and seven guests who had stayed on the same floor of the hotel. One of these hotel guests was admitted into a major public hospital on 24 February and was responsible for the outbreak there affecting at least 88 healthcare workers and 18 medical students. Another major outbreak affecting 237 residents (at the time of writing) in a housing estate was traced back to a patient discharged from the same ward of the public hospital.

The table shows the number of affected healthcare workers in the public hospital and other healthcare facilities, cases in the community, and the total cumulative number of daily reported cases. ${ }^{2}$ At the time of writing, 685 cases had been reported with 16 deaths. The disease, initially affecting mainly healthcare workers, spread rapidly to the community as family members became infected and in turn infected their coworkers and friends. When symptoms developed, they consulted their general practitioners, leading to more healthcare workers developing the disease. It is now known that three of the hotel guests were responsible for the outbreaks in Hanoi, Singapore, and Toronto. There were also smaller clusters arising from individuals having travelled to southern China in the one to two weeks between infection and onset of symptoms. 


\section{Laboratory and pathological findings}

The local experience indicated that the incubation period is between two and 11 days. ${ }^{3}$ All patients presented with fever (temperature $>38^{\circ} \mathrm{C}$ ), chills, and myalgia. Pulmonary infiltrates appeared in chest radiographs early, even before the start of dyspnoea. Symptoms of cough and sputum were not always present. A few patients had diarrhoea. Lymphopenia was the striking feature, with mild thrombocytopenia. Mild liver dysfunction was noted in most cases.

All sputum, blood, and urine cultures were negative for bacterial pathogens. Direct immunofluorescent stains of nasopharyngeal aspirate failed to show the presence of adenovirus; influenza A and B; parainfluenza 1, 2, and 3; and respiratory syncytial virus. Serological examination at one and 14 days did not show increases in titre of antibodies against mycoplasma, chlamydia, or legionella. In some patients the pneumonic infiltration was rapidly progressive, resulting in severe oxygen desaturation requiring assisted ventilation.

A research team in the University of Hong Kong isolated a virus belonging to the family Coronaviridae from two patients. ${ }^{4}$ Using serological and reversetranscriptase polymerase chain reaction specific for this virus, they found 45 out of 50 patients with severe acute respiratory syndrome had evidence of infection with this virus but not in the sera of 80 patients with respiratory or other disease or 200 blood donors. However, reverse-transcriptase polymerase chain reaction is currently only used in research laboratories. At present, the diagnosis of severe acute respiratory syndrome remains a clinical one according to WHO definition. ${ }^{1}$ Diagnostic features include high fever, one or more respiratory symptoms (including cough, shortness of breath, and difficulty breathing), and close contact with a person who has been diagnosed with severe acute respiratory syndrome.

The open lung biopsy of the patient who provided samples for viral culture showed histological changes typical of adult respiratory distress syndrome. ${ }^{3}$ There was diffuse alveolar damage, hyaline membrane formation, and minimal mononuclear cell infiltration. Viral inclusion bodies were not found, although this could have been due to sampling error. The changes in lung tissue suggest that part of the lung damage is due to cytokines induced by the microbial agent. This hypothesis forms the basis of treatment with corticosteroids.

\section{Treatment}

The following empirical treatment has been suggested by the Hospital Authority, Hong Kong, ${ }^{5}$ based initially on the experience with a small number of cases and subsequently confirmed by favourable results in a proportion of patients:

- Broad spectrum antiviral agent-ribavirin $8 \mathrm{mg} / \mathrm{kg}$ every 8 hours intravenously or $1.2 \mathrm{~g}$ every 12 hours orally, with an oral loading dose of $4 \mathrm{~g}$ for those with normal renal function test, for 7-14 days depending on the response and the time of tailing off of corticosteroids

- Hydrocortisone $2 \mathrm{mg} / \mathrm{kg}$ every six hours or $4 \mathrm{mg} / \mathrm{kg}$ every 8 hours intravenously, tail off over one week when there is clear clinical improvement. For severe and rapidly deteriorating cases, methylprednisolone

\section{What is already known on this topic}

Severe acute respiratory syndrome is a form of atypical pneumonia that originated in southern China and spread globally in a few weeks

The clinical picture, empirical treatment, and the possible mode of disease transmission have been described. The agent responsible is probably a novel coronavirus

\section{What this study adds}

This report gives an account of the daily reported number of cases in Hong Kong, showing how the disease has spread rapidly from healthcare workers to the community, and of the drastic measures the government has finally introduced to attempt to control the disease

A vigilant healthcare profession, strict infection control measures, and public education are essential to prevent disease dissemination

Early introduction of quarantine procedures for this disease by health authorities should be considered.

$10 \mathrm{mg} / \mathrm{kg}$ every 24 hours intravenously for two days, and then continue with hydrocortisone as above

- Antibacterial coverage for typical and atypical agents for 7-14 days using drugs such as levofloxacin and macrolides.

Patients should be given antiulcer prophylaxis and monitored for haemoglobin concentration, reticulocyte count, and blood glucose and potassium concentrations. The efficacy of this regimen requires careful assessment.

\section{Precautions}

The most likely route of transmission is by droplets and direct inoculation of secretions on to mucus membrane. The serious outbreak in the public hospital could have been due to the use of nebulised bronchodilator in the index patient, causing atomisation of infected secretion. ${ }^{6}$ Procedures thought to increase the risk of disease transmission include diagnostic sputum induction, bronchoscopy, endotracheal intubation, and airway suction. ${ }^{7}$

The precautions for droplet infection should be instituted. Patients should wear N-95 masks once symptoms develop and be placed immediately in isolation facilities with negative pressure. Healthcare workers should wear similar masks together with head cover, goggles, gowns, and gloves when caring for these patients. Daily and terminal disinfection should be thorough, with careful washing and disinfection of the bed, handrails, bedside tables, floor, and equipment with hypochlorite solution (1000 ppm). For intubated patients, the use of closed suction system is essential to avoid air leak and enhanced disease transmission.

Healthcare workers should have a high index of suspicion if they or family members develop fever and features suggestive of severe acute respiratory syndrome. They should present themselves to hospitals rather than treating themselves at home and putting their family members at risk. For doctors in the 
community, it is advisable to wear a N-95 mask in when seeing any patient with respiratory symptoms. Contacts of proved cases should isolate themselves until the incubation period is over. After contact with patients with respiratory symptoms, careful hand hygiene is necessary, with washing with soap and water.

On 27 March the Department of Health finally announced drastic measures, including vigorous contact tracing and examination, quarantine of contacts in their homes, and closure of all schools and universities. A major hospital has been designated for infected patients. On 1 April all residents from the building in the housing estate where the outbreak occurred were evacuated to a holiday camp. Most public gatherings have been postponed to later dates.

\section{Conclusions}

Severe acute respiratory syndrome is highly infectious and potentially lethal. It caught the medical profession in Hong Kong unaware. The drastic measures introduced by the Hong Kong government, together with intensive education of the public on personal hygiene and the wearing of masks in public places, will, we hope, halt this epidemic. Other health authorities faced with this disease should consider early introduction of quarantine procedures.

Contributors: WCY helped care for the patients. MC-Y collected data for this report and is guarantor.

Competing interests: None declared.

World Health Organization. Severe acute respiratory syndrome (SARS). March 12th 2003. www.who.int/csr/2003 03 12/en

Government of Hong Kong Special Administrative Region Departmen of Health. Atypical pneumonia. www.info.gov.hk/info/infection-c.htm (accessed 7 Apr 2003).

3 Tsang KW, Ho PL, Ooi GC, Yee WK, Wang T, Chan-Yeung M, et al. A cluster of cases of severe acute respiratory syndrome in Hong Kong. $N$ Engl J Med 2003 Apr 1 [epub ahead of print]. http://content.nejm.org cgi/reprint/NEJMoa030666v2.pdf (accessed 7 Apr 2003).

4 Periris JSM, Lai ST, Poon LLM, Guan Y, Yam LYC, Lim W, et al. Coronavirus as a possible cause of severe acute respiratory syndrom. Lancet Online 2003;8 Apr. http://image.thelancet.com/extras/03art3477web. pdf

5 Hospital Authority. Severe acute respiratory syndrome: clinical guidelines (revised 27 Mar 2003). www.ha.org.hk/hesd/nsapi ?MIval=ha view template\&.oroup=PRS\&.Area=CGL (accessed 7 Apr 2003)

6 Lee N, Hui D, Wu A, Chan P, Cameron P, Joynt GM, et al. A major outbreak of severe acute respiratory syndrome in Hong Kong. $N$ Engl J Med 3003; 7 Apr wwwnejm.org

7 Centers for Disease Control and Prevention. Severe acute respiratory syndrome (SARS): information for clinicians. www.cdc.gov/ncidod/sars/ clinicians.htm (accessed 7 Apr 2003)

(Accepted 3 April 2003)

\section{Delayed immunisation and risk of pertussis in infants: unmatched case-control study}

Cameron C Grant, Mavis Roberts, Robert Scragg, Joanna Stewart, Diana Lennon, Denise Kivell, Rodney Ford, Rosalie Menzies

Department of Paediatrics, Faculty of Medicine and Health Sciences, University of Auckland, Private Bag 92019, Auckland, New Zealand

Cameron C Grant senior lecturer

Mavis Roberts project manager

Department of Community Health, Faculty of Medicine and Health

Sciences, University

of Auckland

Robert Scragg

senior lecturer

Joanna Stewar

biostatistician

Child and Youth

Health, South

Auckland Health,

Private Bag 93311,

Otahuhu, Auckland,

New Zealand

Diana Lennon

professor of

community

paediatrics

Denise Kivell

charge nurse

continued over

BMJ 2003;326:852-3
Pertussis remains a severe disease in infants. As about two thirds of infants with pertussis are admitted to hospital, factors that seem to be associated with an increased risk of pertussis may in fact be associated with an increased risk of hospital admission. ${ }^{12}$ The admission rate for pertussis in New Zealand is five to 10 times higher than in England and Wales and the United States. ${ }^{3}$ We determined whether immunisation reduced the risk of admission to hospital for pertussis by comparing infants admitted with pertussis and infants admitted with other acute respiratory illnesses.

\section{Participants, methods, and results}

We performed an unmatched case-control study during the 1995-7 pertussis epidemic in Auckland, New Zealand. Pertussis was defined as cough lasting at least two weeks, with coughing paroxysms, inspiratory "whoop," or vomiting after coughing. The control

Odds ratios (95\% confidence intervals) of catching pertussis associated with delays in giving pertussis vaccine

\begin{tabular}{|c|c|c|c|c|}
\hline \multirow[b]{2}{*}{ Delayed immunisation } & \multicolumn{2}{|c|}{ No/total (\%) of participating infants with delayed dose } & \multicolumn{2}{|c|}{ Adjustment for } \\
\hline & Pertussis cases $(\mathrm{n}=97)$ & Hospital controls $(\mathrm{n}=98)$ & Age only & 0ther variables* \\
\hline First dose of DTPH† & $13 / 70(19)$ & $10 / 71(14)$ & $2.13(0.77$ to 6.20$)$ & 2.23 (0.64 to 8.67$)$ \\
\hline Second dose of DTPH† & $10 / 25(40)$ & 18/49 (37) & $1.60(0.53$ to 4.94$)$ & 2.37 (0.59 to 10.40$)$ \\
\hline Third dose of DTPH† & $9 / 13(69)$ & $9 / 26(35)$ & 4.25 (1.07 to 19.54$)$ & 6.09 (1.00 to 49.64$)$ \\
\hline Any immunisation & $21 / 70(30)$ & 23/71 (33) & 2.66 (1.02 to 7.71$)$ & 4.50 (1.22 to 20.94$)$ \\
\hline
\end{tabular}

*Multivariate analyses included variables describing infants' age, social deprivation, and crowding in households, and, in the analysis of delay for any immunisation, maternal education.

tDTPH=Diphtheria-tetanus-pertussis-haemophilus type B whole cell vaccine (Tetramune; Wyeth Lederle, United States). group consisted of 98 infants admitted to hospital with a coughing illness who were culture negative for Bordetella pertussis and had no $B$ pertussis DNA detected in their nasopharyngeal sample after amplification by polymerase chain reaction. We interviewed each infant's care giver and determined written confirmation of the infant's immunisation status from his or her health record book or the family doctor's records.

In New Zealand, immunisations are scheduled at age 6 weeks, 3 months, and 5 months. An immunisation was delayed if it had not been received within 30 days of its first being due. ${ }^{4}$ We used logistic regression to calculate odds ratios and 95\% confidence intervals to determine the risk of pertussis associated with delayed immunisation. We defined socioeconomic status by the occupation of the household's main income earner. We measured social deprivation by using the 1996 New Zealand social deprivation index. 